Artículos de investigación científica y tecnológica / scientific and tecnological research papers

\title{
Relación entre la presencia de surcos radiculares y las variaciones anatómicas de primeros premolares inferiores de una subpoblación colombiana, según Vertucci
}

\author{
Relationship between the presence of radicular grooves and the anatomical \\ variations according to Vertucci of mandibular first premolars of a Colombian \\ subpopulation
}

Lucía Isabel Franco Márquez ${ }^{(\mathbb{D})}$, Iván Darío Gómez Hernández ${ }^{1}\left(\mathbb{D}\right.$, Andrea Johanna Almario Barrera ${ }^{2} \mathbb{D}^{\mathrm{D}}$, Martha Lucely Duarte Monsalve $^{3 \text { (D) Jaime Omar Moreno Monsalve }}{ }^{4}$ (D)

Citación: Franco-Márquez LI, Gómez-Hernández ID, Almario-Barrera AJ, Moreno JO. Relación entre la presencia de surcos radiculares y las variaciones anatómicas de primeros premolares inferiores de una subpoblación colombiana, según Vertucci. Ustasalud 2021; 20: 7-11.

Licencia Creative Commons

(c) (1) (9) lo tanto, los lectores pueden acceder libremente a los artículos en su formato .pdf, igualmente podrán descargarlos y difundirlos; sin embargo no podrán modificarlos o alterarlos, adicionalmente se debe reconocer la autoría de las personas que figuran en las publicaciones, pero estas no podrán ser comercializadas.

\section{Resumen}

El objetivo de este estudio es reconocer surcos radiculares (SR) en la anatomía externa radicular conforme a la clasificación de Vertucci en primeros premolares inferiores de una subpoblación colombiana.

Mediante evaluación imagenológica realizada con tomografía computarizada de haz cónico (CBCT) en tres planos (coronal, sagital y axial), a una muestra de 140 primeros premolares inferiores humanos, se realizó la configuración anatómica de los conductos radiculares de acuerdo con la clasificación de Vertucci. A partir de los cortes axiales de las imágenes de CBCT, fueron reconocidos los $S R$.

Se estableció que la tipología I según la clasificación de Vertucci fue la que presentó mayor frecuencia $72,14 \%$ (101/140), seguida por la tipo $V$ en $19,28 \%$ (27/140). De igual manera, la presencia de surcos radiculares $(S R)$ correspondió al 30\% (42/140).

Los resultados reportan gran variabilidad anatómica en el primer premolar inferior, lo que sustenta la necesidad de estudiar con detalle la anatomía dental y con ello orientar las estrategias y protocolos de manejo y atención que garanticen el éxito del tratamiento endodóntico.

Palabras claves: Primer premolar inferior, tomografía compuratizada de haz cónico, surco radicular, configuración de canal radicular, anatomia dental.

\section{Abstract}

The purpose of this research was to identify the presence of radicular grooves $(S R)$ in the external root anatomy and its relationship with the Vertucci classification in mandibular first premolar of a Colombian subpopulation.

Images were analyzed using cone beam computed tomography (CBCT) in three planes (coronal, sagittal and axial), of 140 human mandibular first premolar, and the anatomical configuration of the root canals was determined according to the classification of Vertucci. Subsequently, the SRs of these pieces were recognized in the axial sections of the $\mathrm{CBCT}$ images.

It was established that typology I according to the Vertucci classification was the one with the highest frequency $72.14 \%$ (101/140), followed by type $V$ in $19.28 \%$ (27/140). Similarly, the presence of root grooves $(S R)$ corresponded to $30 \%(42 / 140)$.

With this study, a large percentage of anatomical variations in mandibular first premolar of a Colombian population was evidenced, this reinforces the need to know in detail the dental anatomy to establish more effective strategies and protocols and thus achieve a successful endodontic treatment at long term.

Keywords: Mandibular first premolar, computed tomography, radicular groove, root canal configuration, dental anatomy.
1 Estudiante Facultad Odontología, Universidad Santo Tomás, Bucaramanga.

2 Odontólogo, docente Facultad de Odontología, Universidad Santo Tomás, Bucaramanga.

3 Magíster en Odontología, docente Facultad de Odontología, Universidad Santo Tomás, Bucaramanga.

4 Ph.D en Odontología, docente Facultad de Odontología, Universidad Santo Tomás, Bucaramanga.

Autor de correspondencia: Jaime Omar Moreno Monsalve Correo electrónico: jaime.moreno@ustabuca.edu.co Recibido para publicación 15 de julio de 2020 Aceptado para publicación: 4 de marzo de 2021 


\section{INTRODUCCIÓN}

Uno de los propósitos del tratamiento endodóntico está orientado al manejo de la periodontitis apical (PA) [1]. Así, la persistencia de la PA postratamiento endodóntico se constituye en un fracaso clínico el cual puede ser causado por la persistencia microbiológica en las superficies anatómicas complejas del sistema de canales radiculares (SCR) en áreas de difícil acceso [2]. A pesar de la aparición de instrumentos y dispositivos endodónticos en el mercado con mejores capacidades tecnológicas, estos no logran eliminar por completo el biofilm que se acumula en áreas de difícil acceso, tales como canales ovalados, istmos, ramificaciones, concavidades, deltas apicales, los cuales siguen siendo un punto crítico durante la preparación química y mecánica, dado que allí se facilita la permanencia de remanentes tisulares y microbianos [3].

Usualmente, dientes con una raíz tienen un canal intrarradicular, sin embargo, se puede encontrar en ciertos dientes como los incisivos y premolares inferiores dos o más canales [4]. Además, en primeros premolares inferiores se han detectado invaginaciones o surcos verticales, principalmente en el tercio medio radicular de las caras proximal-lingual [5]. Asimismo, se han descrito surcos o depresiones en la superficie mesial y distal [6].

Adicionalmente, cabe destacar que en los primeros premolares inferiores se han asociado la anatomía externa (surcos) y la interna (número de canales) [7]. Por lo tanto, es fundamental reconocer de forma integral la morfología externa e interna en los diferentes grupos dentales y sus variaciones para garantizar una terapia endodóntica exitosa [8].

Por otra parte, se han documentado fallas en el rendimiento diagnóstico a partir de imágenes radiográficas convencionales, que tradicionalmente se han empleado para el reconocimiento de estos surcos, ya que en ocasiones se evidencia una línea radiolúcida vertical adentro de la raíz y paralela al ligamento periodontal, más aún, puede observarse como una zona radiolúcida similar a una lágrima o se puede confundir con otro canal radicular $[9,10,11]$. Ante estas interpretaciones difusas, se han hecho investigaciones orientadas a proponer nuevas estrategias diagnósticas como lo hizo Robinson y col, quienes en su estudio en 2002 documentaron la capacidad de la tomografía dental computarizada para interpretar la anatomía externa e interna de premolares inferiores [9].

En síntesis, la evidencia científica disponible a la fecha carece de información relacionada con los surcos radiculares y la morfología del SCR, en ciertas poblaciones, dentro de ellas la suramericana [7]. Así, este trabajo propone una investigación orientada a reconocer la presencia de surcos radiculares en la anatomía externa y su relación con la clasificación de Vertucci en primeros premolares inferiores en una población colombiana, haciendo uso de la tomografía computarizada de haz cónico (CBCT).

\section{MATERIALES Y MÉTODOS}

Este estudio fue aprobado por el Comité de Investigaciones de la Facultad de Odontología (Universidad Santo Tomás, Bucaramanga, Colombia) bajo la Resolución 08430.

Se utilizaron 140 primeros premolares inferiores humanos, extraídos, obtenidos en el Banco de Piezas Dentarias de la Universidad Santo Tomás, Bucaramanga, Colombia, previa firma del consentimiento informado de donación. El género y edad de los pacientes fue desconocido y todos los dientes fueron extraídos por razones no relacionadas con este estudio.

Los criterios de inclusión fueron los siguientes: dientes primeros premolares inferiores con ápices formados, sin reabsorciones o fracturas, sin tratamientos endodónticos previos.

Los especímenes fueron enumerados del 1 al 140, y se formaron 10 grupos de 14 dientes cada uno, montados en una base doble de cera rosada para bases (BonDent), para ajustar cada grupo al campo de visión $(F O V)(8 \times 9)$, en seguida, las imágenes CBCT de los dientes fueron efectuadas con un tomógrafo marca CS8100 3D family, con resolución de 150 micras.

Las imágenes CBCT fueron visualizadas con los programas Carestream y CS 3D Imaging Software en una habitación oscura en los tres planos de reconstrucción (axial, coronal, sagital); se registró el número de conductos para cada diente y se identificó la tipología basados en la clasificación de Vertucci. 
A continuación, el total de los 140 dientes fueron examinados, empleando el corte axial para cada diente en las imágenes CBCT para comprobar la presencia de $S R$. La información recolectada en el estudio se consignó en una base de datos en Excel con doble sistematización para minimizar errores de digitación, se verificó la calidad de la digitación mediante la rutina validate del paquete Epilnfo (versión 7, Miami, EE.UU) y, finalmente, se exportó al paquete estadístico STATA.

La reconstrucción y análisis de la imágenes CBCT fue ejecutada y repetida tres veces por un solo observador.

\section{RESULTADOS}

La clasificación Vertucci observada con mayor frecuencia fue la tipo $I 72,1 \%(101 / 140)$, seguida de la tipo $V 19,3 \%$ (27/140) (Tabla 1, Fig. 2). De igual manera, se distinguió presencia de $S R$ en $30 \%(42 / 140)$ (Tabla 2 y Figuras 1 y 3 ).

Tabla 1. Distribución frecuencias de la configuración Vertucci en primeros premolares inferiores

\begin{tabular}{cc}
\hline Configuración Vertucci & $\mathbf{n}(\%)$ \\
\hline Tipo I & $101(72,1)$ \\
\hline Tipo III & $7(5)$ \\
\hline Tipo V & $27(19,3)$ \\
\hline Tipo VII & $1(0,7)$ \\
\hline No Vertucci & $4(2,9)$ \\
TOTAL & $140(100)$ \\
\hline
\end{tabular}

Tabla 2. Distribución frecuencias de $S R$ en primeros premolares inferiores.

\begin{tabular}{cc}
\hline Frecuencia de $S R$ & $\mathbf{n}(\%)$ \\
\hline SÍ & $42(30 \%)$ \\
NO & $98(70 \%)$ \\
\hline
\end{tabular}

Al relacionar la presencia de $S R$ con la configuración de Vertucci, se encontró mayoría 61,9\% (26/42) para la tipología $V$, seguida por la tipo $I I I$ con $16,6 \%$ (7/42) (Tabla 3). Por otra parte, el 9,5\% (4/42) dientes con $S R$, fueron tipo $I$ y un diente sin $S R$, mostró tipología $V$ (Tabla 3 y Figura 2).

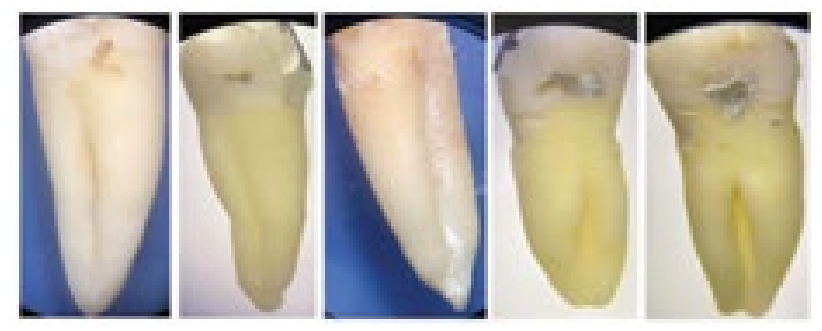

Figura 1. Presencia de $S R$ en primer premolar inferior.

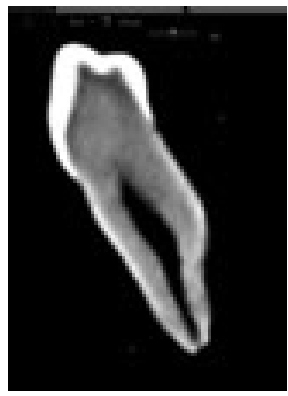

Tipo I: 1

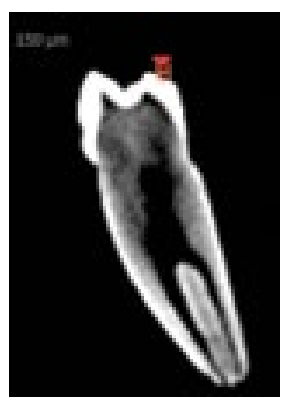

Tipo III: 1-2-1

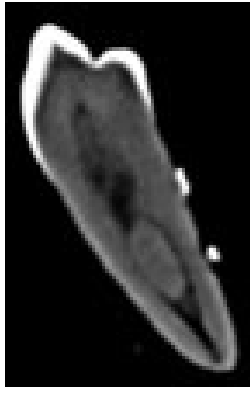

Tipo V: 1-2

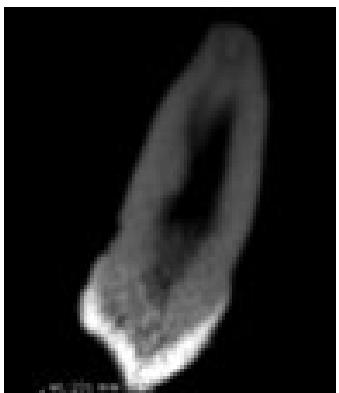

Tipo VII: 1-2-1-2
Figura 2. Tipos de clasificación de Vertucci observados: corte $V$ - $L$ tomografía computarizada de haz cónico.

Tabla 3. Relación presencia de $S R$ con la frecuencia de configuración Vertucci en primeros premolares inferiores.

\begin{tabular}{ccccccc}
\hline & I & III & V & VII & NO Vertucci & TOTAL \\
\hline Presencia de $S R$ & & & & & & \\
SÍ & $4(9,5 \%)$ & $7(16,6 \%)$ & $26(61,9 \%)$ & $1(2,3 \%)$ & $4(9,5 \%)$ & 42 \\
NO & 97 & 0 & 1 & 0 & 0 & 98 \\
TOTAL & 101 & 7 & 27 & 1 & 4 & 140 \\
\hline
\end{tabular}



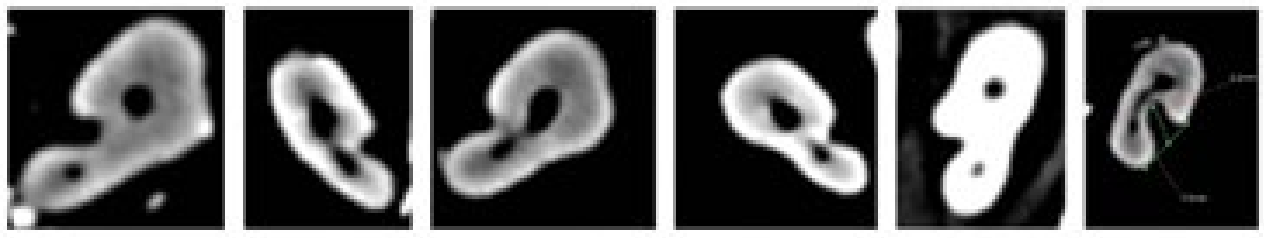

Figura 3. $S R$ vistos en corte axial CBCT.

\section{DISCUSIÓN}

Este estudio ex vivo evaluó la relación entre el hallazgo de $S R$ y las variaciones anatómicas del SCR de acuerdo con la clasificación Vertucci, en primeros premolares inferiores mediante el uso de CBCT como herramienta de apoyo en el diagnóstico. La CBCT es una técnica no invasiva que le permite al clínico hacer una valoración de la anatomía dental interna y externa a partir de la reconstrucción en tres dimensiones. El tamaño del voxel empleado fue de 150 micrones de resolución, favoreciendo así el reconocimiento de las variaciones anatómicas radiculares externas e internas [12].

En estudios preliminares, la frecuencia de $S R$ en este tipo de dientes ha sido del 9,0\% [9] y 17,6\% [13], estudios posteriores en China reportaron 24,0\% [14], $27,8 \%$ [8], 40,9\% [11] y, 56,2\% [15]; como es observado, se tienen promedios variables entre $9,0 \%$ y $56,2 \%$; en nuestro estudio, la frecuencia de $S R$, fue $30,0 \%$ $(42 / 140)$ similar al estudio de Liu y colaboradores en 2013, que reportaron $27,8 \%$ [8].

Con respecto a la relación entre $S R$ y la configuración de Vertucci en los 42 dientes que lo presentaron, la tipología $V$, fue la más frecuente, con el $61,9 \%$ (26/42) semejante del estudio de Bochetti y colaboradores 2017, que señalaron un 58,6\% (41/70) de configuración tipo $V$. Debemos señalar, que en solo un diente con configuración tipo $V$, no se observó $S R$.

La prevalencia de $S R$ en dientes con tipología $I$ de Vertucci fue 2,9\% (4/140) en el presente estudio, semejante al estudio de Shemesh y colaboradores 2020, que encontraron 3\% (44/1432) de Vertucci tipo I [16].

\section{CONCLUSIÓN}

Debemos destacar del presente estudio in vitro, que sí hay una relación directa entre la presencia de $S R$ y las diferentes tipologías observadas. Estos hallazgos indican que las variaciones anatómicas del SCR en los primeros premolares inferiores analizados tienden a presentarse con mayor frecuencia cuando se observan $S R$ en su superficie externa.

Todo clínico debe considerar la complejidad anatómica que potencialmente presentan los primeros premolares inferiores con SR antes de realizar un tratamiento endodóntico, y tomar así los recaudos necesarios para localizar el conducto lingual extra, y con un protocolo viable disminuir la prevalencia de PA.

\section{REFERENCIAS}

[1] Siqueira JF Jr, Rôças IN, Ricucci D, Hülsmann M. Causes and management of post-treatment apical periodontitis. Br Dent J. 2014;216(6):305-312. doi:10.1038/ sj.bdj.2014.200.

[2] Ricucci D, Siqueira, JF Jr. Anatomic and Microbiologic Challenges to Achieving Success with Endodontic Treatment: A Case Report. J Endod. 2008;34(10):1249-1254. doi:10.1016/j.joen.2008.07.002.

[3] Lopes HP, Siqueira JF Jr. Endodoncia: Biología y Técnica. 4a. Ed. São Paulo: Quintessence Editora, 2020. ISBN 978-85-7889-172-5.

[4] Ordinola-Zapata R, Bramante CM, Villas-Boas $\mathrm{MH}$, Cavenago BC, Duarte MH, Versiani MA. Morphologic micro-computed tomography analysis of mandibular premolars with three root canals. J Endod. 2013;39(9):11301135. doi:10.1016/j.joen.2013.02.007.

[5] Lu TY, Yang SF, Pai SF. Complicated root canal morphology of mandibular first premolar in a Chinese population using the cross section method. J Endod. 2006;32(10):932-936. doi:10.1016/j.joen.2006.04.008.

[6] Cleghorn BM, Christie WH, Dong CC. The root and root canal morphology of the human mandibular first premolar: a literature review. J Endod. 2007;33(5):509516. doi:10.1016/j.joen.2006.12.004.

[7] Boschetti E, Silva-Sousa YTC, Mazzi-Chaves JF, Leoni GB, Versiani MA, Pécora JD et al. Micro-CT Evaluation 
of Root and Canal Morphology of Mandibular First Premolars with Radicular Grooves. Braz Dent J. 2017 28(5):597-603. doi:10.1590/0103-6440201601784.

[8] Liu N, Li X, Liu N et al. A micro-computed tomography study of the root canal morphology of the mandibular first premolar in a population from southwestern China. Clin Oral Investig. 2013;17(3):999-1007. doi:10.1007/ s00784-012-0778-1.

[9] Robinson S, Czerny C, Gahleitner A, Bernhart T, Kainberger FM. Dental CT evaluation of mandibular first premolar root configurations and canal variations. Oral Surg Oral Med Oral Pathol Oral Radiol Endod. 2002;93(3):328-332. doi:10.1067/moe.2002.120055.

[10] Simon JH, Dogan H, Ceresa LM, Silver GK. The radicular groove: its potential clinical significance. J Endod. 2000;26(5):295-298. doi:10.1097/00004770-200005000-00012.

[11] Chen J, Li X, Su Y et al. A micro-computed tomography study of the relationship between radicular grooves and root canal morphology in mandibular first premolars. Clin Oral Investig. 2015;19(2):329-334. doi:10.1007/ s00784-014-1242-1.

[12] Patel S, Dawood A, Ford TP, Whaites E. The potential applications of cone beam computed tomography in the management of endodontic problems. Int Endod J. 2007;40(10):818-830. doi:10.1111 /j.1365-2591.2007.01299.
[13] Awawdeh LA, Al-Qudah AA. Root form and canal morphology of mandibular premolars in a Jordanian population. Int Endod J. 2008;41(3):240-248. doi:10.11 11/j.1365-2591.2007.01348.

[14] Fan B, Yang J, Gutmann JL, Fan M. Root canal systems in mandibular first premolars with C-shaped root configurations. Part I: Microcomputed tomography mapping of the radicular groove and associated root canal cross-sections. J Endod. 2008;34(11):1337-1341. doi:10.1016/j. joen.2008.08.006.

[15] Gu Y, Zhang Y, Liao Z. Root and canal morphology of mandibular first premolars with radicular grooves. Arch Oral Biol. 2013;58(11):1609-1617. doi:10.1016/j. archoralbio.2013.07.014.

[16] Shemesh A, Lalum E, Ben Itzhak J, Levy DH, Lvovsky A, Levinson O, Solomonov M. Radicular Grooves and Complex Root Morphologies of Mandibular Premolars Among Israeli Population. J Endod. 2020;46(9):12411247. doi: $10.1016 /$ j.joen.2020.05.013. Epub 2020 Jun 10. PMID: 32531312.

\section{Correo electrónico de los autores:}

Lucia Isabel Franco Márquez: lucia.franco@ustabuca.edu.co Iván Darío Gómez Hernández: ivan.gomez02@ustabuca.edu.co Martha Lucely Duarte Monsalve: martha.duarte@ustabuca.edu.co Jaime Omar Moreno Monsalve: jaime.moreno@ustabuca.edu.co Andrea Johanna Almario Barrera: andrea.almario@ustabuca.edu.co 